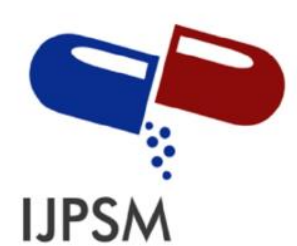

Nikam Supriya S. et al, International Journal of Pharmaceutical Sciences and Medicine (IJPSM),

Vol.6 Issue. 7, July- 2021, pg. 61-80

ISSN: 2519-9889

Impact Factor: 3.426

\title{
POTENTIAL OF NANOSTRUCTURED LIPID CARRIERS IN NOSE TO BRAIN TARGETING FOR ALZHEIMER'S DISEASE
}

\author{
Nikam Supriya S. ${ }^{*}$; Watode Ankita B. ${ }^{1}$; Phadatare Priya P. $^{2}$; Kalyani Kayande ${ }^{3}$ \\ ${ }^{1}$ Sinhgad Institute of Pharmacy Narhe, Pune, Maharashtra, INDIA \\ *Email id-sdeshmukh3105@gmail.com \\ DOI: 10.47760/ijpsm.2021.v06i07.006
}

\begin{abstract}
Due to the number of disorders associated with oral, parenteral, rectal and other routes of administration, Scientists are increasingly interested in exploring the potential nasal uses of various drugs. Intranasal drug delivery systems are commonly known to treat ailments such as colds, coughs, and rhinitis. This review therefore focuses on new research on nanostructured lipid transporters for nasal brain targets that are beneficial for various neurodegenerative disorders. Here highlighted one is Alzheimer disorder. The incorporation of drugs into nanoparticles selects drug delivery from the nose to the brain due to high drug absorption through the nasal mucosa and many benefits of brain-targeted drug delivery such as avoidance of BBB could be a talent approach. This is achieved by pathway mechanism. NLC as a lipid-containing drug delivery system, is an established method for pharmaceutical preparation for all major types of DDS in Nano science. NLC is a new type of DDS, stable in various environments and capable of forming centralized dispersions. This article covers various processes of NLC production/ preparation and its applications with current advances. NLC can increase drug delivery to target organs, alter the pharmacokinetics of drug carriers to enhance therapeutic effects and reduce harmful side effects.
\end{abstract}

Keywords: Alzheimer's disease, Nasal drug delivery system, Nanostructured Lipid Carrier

\section{Introduction ${ }^{[1][2][3][4][5][6][7][8]}$}

Proper delivery to the brain is a main motive for many drugs and dare for pharmaceutical researchers. The low drug distribution of the brain is surely due to the presence of main barrier that is the blood-brain barrier. Drugs with lipophilic molecules, a partition coefficient (Log P) of 1.5 to 2.7, and a molecular weight of less than 600 daltons (Da) can enter the brain barrier. A more active and functional definition recognizes the attached endothelial structure as an integral part of the BBB. There is not only presence of endothelial cells but also it is composed of astrocytes, 


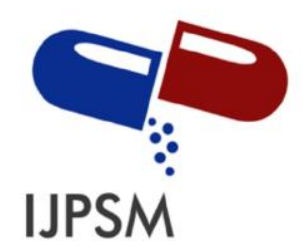

Nikam Supriya S. et al, International Journal of Pharmaceutical Sciences and Medicine (IJPSM), Vol.6 Issue. 7, July- 2021, pg. 61-80

ISSN: 2519-9889

Impact Factor: 3.426

pericytes, neurons, and extracellular matrix. This established neurovascular unit is important for protecting underlying brain cells and maintaining the CNS homeostasis, necessary for coordinated and stable neurons activity. BBB protects brain against polar drugs, peptides and protein substances. This is particularly beneficial for drugs that do not easily cross the BBB due to their physicochemical properties. The vascular system of the central nervous system is characterized by the presence of blood brain barrier, which separates the interstitial fluid in the brain from circulating blood. BBB not only protects the brain from blood drugs that can alter nerve function, but also controls the entry and exit of substances to provide the brain with the necessary nutrients and maintain the proper migration of important ingredients. The cells of the brain's capillary epithelium are held together by complex tight junctions. These tight junctions completely surround each endothelial cell, connecting both adjacent cells and adjacent borders of the same cell. Each cerebral capillary is composed of two lipid membranes $300 \mathrm{~nm}$ distant from the endothelial cytoplasm, with the inner space facing the blood and the outer space facing brain. Unbearable underlying conditions such as Alzheimer's disease and Parkinson's disease partially or completely include the central nervous system and affect neurochemical levels and neural pathways in brain. To treat these conditions, the drug must increase access to the brain. Also, to control pain, the drug must reach the brain, where various receptors are stimulated by therapeutic compounds. Effective penetration of the drug into the brain is also the key to effective treatment of the brain tumors. Indeed, many powerful therapies have been abandoned solely because of the inability to successfully implement CNS. Therefore, it is beneficial to use another non-invasive targeted brain delivery method that bypasses the BBB.

Many scientists have tried to select the drug from the nose to the brain to bypass BBB. The benefits of nasal nose to brain formulation have been reported in terms of safety, first-pass metabolism, non-invasiveness and compatible ${ }^{2}$ The evidence's suggests nasal delivery has been shown to be useful for the systemic delivery of drugs such as low molecular weight polarizers, peptides and proteins which are not readily available by other deliveries. A variety of nasal products are under development as they become more effective when administered intranasally. We also appreciate the possibility of using this distribution of pathways to achieve the goals of brain and CSF normalization.It is associated with the management of emergencies. Various research studies in the $20^{\text {th }}$ century evaluate that the nasal compartment is an important for viruses to enter the brain. Polio was the first virus to be transmitted by this way, and these studies also found polioviruses presence in the cerebrospinal fluid and nervous system and also in the lymphatic system of the olfactory mucosa. Subsequently, there was further evidence supporting the direct entry of the virus into the brain via the nose. In nasal compartment, the olfactory epithelium is the only aperture of the central nervous system where dendritic processes of the nerve cells are in direct contact with the environment above the nasal compartment. In the olfactory bulb there is synaptic glomeruli is present so that through the ethmoid disc hole of it the bundle of axon is passed into it. The nose has potential to deliver drugs directly to the central nervous system. 


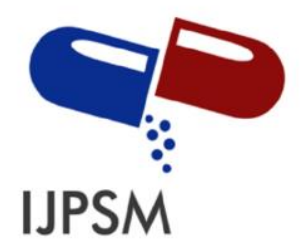

Nikam Supriya S. et al, International Journal of Pharmaceutical Sciences and Medicine (IJPSM), Vol.6 Issue. 7, July- 2021, pg. 61-80

ISSN: 2519-9889

Impact Factor: 3.426

Currently, there is significant evidence in the literature that drugs administered intranasally in animals and humans can be delivered directly to the CNS.

The maximum number of approved combination methods that bypass the BBB allows the drug to achieve its goals in the CNS and includes invasive methods such as direct injections, transient but beneficial BBB interruptions, etc. Returns do not always outweigh risk. After that, drug-based drug design was implemented. Currently, nanotechnology-based methods are being developed as drug delivery processes, regardless of the nature of the molecule. This is generally to surround the molecule and hide the possibility of physicochemical defects. To overcome the limitations of nasal drug delivery, nanoparticles have applications in many areas such as pharmaceuticals, medicine and cosmetics also ignore BBB.

This review examines the use of intranasal lipid-based nanosystem as a promising strategy to improve the management of neurological disorders, a wide range of disorders affecting CNS. This article begins with a brief explanation of the importance of nanotechnology in delivering nasal passages to the brain for the treatment of Alzheimer's disease. An explanation of the anatomy of the nose and subsequent dosing requirements is provided. The final section describes the function of lipid-based nanosystem for drug delivery and reports the most relevant studies involving intranasal delivery to improve disorders related to CNS.

\section{Alzheimer's Disease ${ }^{[9][10][11][12][13]}$}

According to a report by the Alzheimer's disease Association (2017), Alzheimer's disease (AD) is the sixth leading cause of death in the United States, affecting nearly 5.5 million people. In 2017, the cost of treating AD in the United States was estimated to have reached $\$ 259$ billion, and this statistic shows how devastating the disease is in the United States, which is very similar around the world. Additionally, treatment is limited to some common oral medications that have been approved by the FDA. Visually, it is limited to mild to moderate AD. The therapeutic efficacy of this drug is influenced by the presence of BBB and by reduced concentrations in the brain due to poor brain permeability.

By 2050, the financial burden imposed by AD is expected to exceed $\$ 230$ billion and reach $\$ 1.1$ trillion. Given the clinical and financial burden associated with $\mathrm{AD}$, the key need is to identify new pathogenesis tools and new therapeutic targets. The title AD is named after Dr. Alois Alzheimer, a German physician, who observed changes in the brain tissue of a woman who died of an unusual mental illness in 1907. Loss of neuronal cells in brain regions essential for memory and other psychic abilities. Alzheimer's disease causes a gradual but slow loss of a person's mental, behavioral and functional abilities. Various causes of AD include amyloid precursor protein (APP) accumulation, $\mathrm{A} \beta$ peptide accumulation, and neuronal damage. $\mathrm{AD}$ is associated with three major structural changes in the brain. Diffuse loss of neurons, deposition of intracellular proteins called neurofibrillary tangles (NFTs) composed 


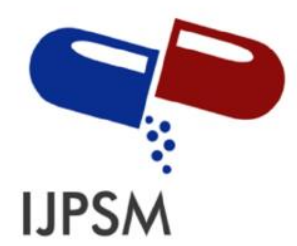

Nikam Supriya S. et al, International Journal of Pharmaceutical Sciences and Medicine (IJPSM), Vol.6 Issue. 7, July- 2021, pg. 61-80

ISSN: 2519-9889

Impact Factor: 3.426

of highly phosphorylated tau protein, and deposition of extracellular proteins called amyloid ( $\mathrm{Ab}$ ) or amyloid plaque. Dystrophy neuritis, changes in gene expression between $\mathrm{AD}$ and normal brain. This analysis is one of the hypothetically powerful approaches to identify the genes involved in pathogenesis. Current imaging methods have the potential to be intriguing in determining whether an individual has $\mathrm{AD}$ or other forms of dementia. These include positron emission tomography (PET), single photon emission tomography and functional magnetic resonance imaging (MRI) to measure functional brain activity, computed tomography, spectroscopy and spectroscopy. Hydrogen magnetic resonance (1H MRS) and resonance spectroscopy (MRS) to measure the chemical composition of magnetic resonance imaging for the anatomy and magnetism of the brain. These methods have been shown to aid proactive diagnosis even in individuals at risk and to distinguish $\mathrm{AD}$ from other causes of dementia such as vascular dementia and brain dementia.

Drug Administration (FDA), includes five drugs that are used to treat the cognitive manifestations of AD AChEIsrivastigmine (Exelon), galantamine (Razadyne, Reminyl), tacrine (Cognex), and donepezil (Aricept) and NMDA receptor antagonist-memantine (Namenda) that target symptoms at its best. Each drug acts in a different manner to delay the breakdown of Ach (a chemical in the brain important for memory. AD is related with insufficient levels of important neurotransmitter. Tacrine (Cognex) is not often prescribed due to its serious side effects (liver damage) and in general, Reminyl, Exelon and Aricept are most active when treatment is initiated in the early stages but Memantine (Namenda) is the only drug shown to be active for the later stages of the disease. They have all been shown to modestly measured the progression of cognitive signs and decrease challenging behaviour's in some people also at least half of the people who take these drugs do not respond to them. These present treatment strategies only delay the progression of symptoms associated with $\mathrm{AD}$. Researchers reported different lipid-based formulations to progress solubility and stability, to increase oral bioavailability, for topical delivery, in treatment of cancer, in treatment of inflammation and infections, and other diseases. The nasal route is estimated by various researchers for drug delivery of drugs into the brain.

\section{Nasal drug delivery system comparison between Oral, Parenteral and Transdermal DDS ${ }^{[14]}$}

Nasal DDS is a novel promising approach of drug delivery system in which, drug is targeted in nose to brain. It is irreplaceable approach to target the drug direct from nose to brain bypassing the BBB so that the nasal drug delivery system comparison with oral, parenteral and transdermal Drug delivery system. The comparison of Nasal drug delivery system with oral, parenteral and transdermal drug delivery system given in table1.

\section{Nasal Administration ${ }^{[15]}$}

The nasal passage involves the absorption of drugs into the nasal mucosa for local or systemic action or to target the brain. Therefore, the term "intranasal" is used to refer to the absorption of the drug through the nasal mucosa acting 


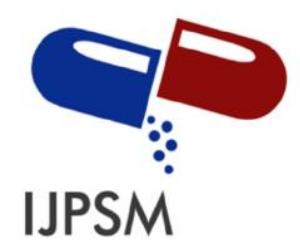

Nikam Supriya S. et al, International Journal of Pharmaceutical Sciences and Medicine (IJPSM), Vol.6 Issue. 7, July- 2021, pg. 61-80

ISSN: 2519-9889

Impact Factor: 3.426

elsewhere, and "nose" refers to the local action, so the standard term refers only to the "nose". It is important to note that it includes nasal and nasal administration. Compared with other routes, using the nasal route has more advantages. For example, it is non-invasive and easy to use. Application and painless compared to parenteral and rectal routes, the ability to deliver drugs directly Upon entering the bloodstream, it escapes the gastrointestinal inactivation and rapid hepatic metabolism found in some oral drugs; especially the large surface area of the nasal mucosa, the high systemic absorption of the drug due to its permeability and vascular distribution; quick effect. It has better bioavailability than the Oral initiator; Ability to use drugs.

Table 1. Comparison with oral, parenteral and transdermal drug delivery system

\begin{tabular}{l|ll|l|l|l|}
\hline Parameters & Yes & No & Yes & Yes \\
\hline Higher Plasma drug levels & & Yes & No & No & No \\
\hline BBB and CSF bypass & & & & \\
\hline Rapid onset & Yes & No & Yes & Yes \\
\hline Pain at the site of administration & No & No & Yes & No \\
\hline Mucosal irritation & No & Yes & No & Yes \\
\hline Systemic activity & Yes & No & Yes & Yes \\
\hline Self-administration & Yes & Yes & No & Yes \\
\hline Patient compliance & High & High & Low & Low \\
\hline Drug degradation & No & High & No & Low \\
\hline Hepatic first pass metabolism & No & Yes & No & No \\
\hline Targeted delivery & Yes & No & Yes & Yes \\
\hline & & & & \\
\hline
\end{tabular}




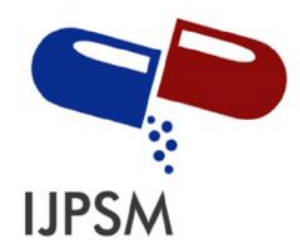

Nikam Supriya S. et al, International Journal of Pharmaceutical Sciences and Medicine (IJPSM), Vol.6 Issue. 7, July- 2021, pg. 61-80

\section{Anatomy of Nose $e^{[2][6][7][16]}$}

The nose has a complex structure. As shown in figure 1, the nasal cavity is $12-14 \mathrm{~cm}$ deep and extends from the nasal vestibule to the nasopharynx, having three main areas; Vestibular, respiratory and olfactory regions. Nose has a volume of $16-19 \mathrm{~cm}^{2}$, an area of about $180 \mathrm{~cm}^{2}$, two cavities separated by septum, nostrils. The vestibular area, which often contains long hairs, is responsible for filtering suspended particles located in the opening of the nasal cavity.

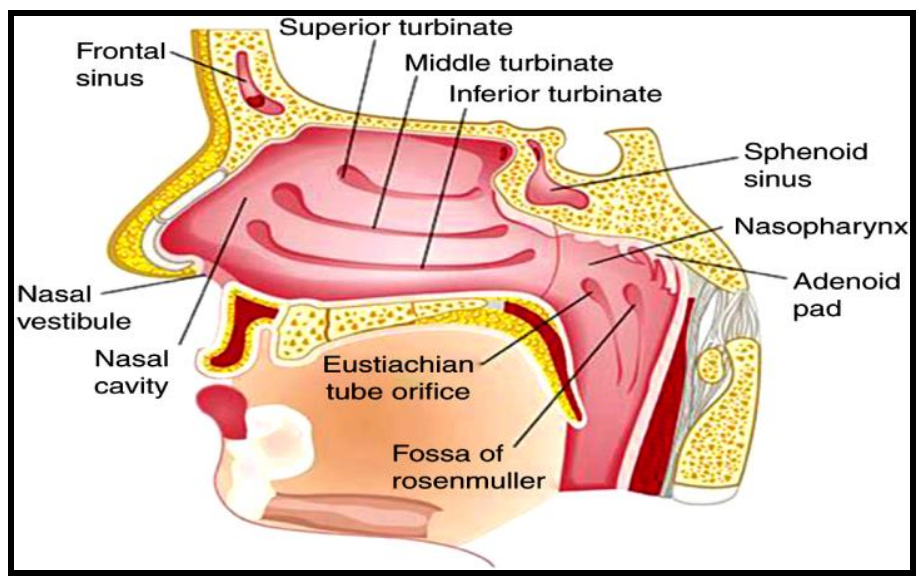

Figure 1. Anatomy of the human nasal cavity.

The vestibular area is not involved in the absorptive function. It is the anterior outer region leading to the nasal cavity. The respiratory epithelium is composed of ciliated and non-ciliated cylindrical cells, basal cells, and mucus-secreting goblet cells. These cells promote active transport and help prevent mucous membrane dryness by retaining water. The respiratory tract is involved in drug absorption, and the respiratory tract in humans is approximately $160 \mathrm{~cm}^{2}$. It is the largest of the three and its biliary epithelium acts as a system for removing particles deposited in the mucous layer. The third zone is the olfactory zone, which contains olfactory receptor cells, basal cells, and olfactory cells, and is made up of thick connective tissue and the stroma in which the olfactory epithelium is located. The thickness of the nasal mucosa varies from 2 to $4 \mathrm{~mm}$. Epithelial cells line the nasal cavity and are covered with a $5 \mu \mathrm{m}$ thick layer of mucus to trap unwanted particles. Mucous secretions contain 95\%, 2\%, 1\%, 1\% (albumin, immunoglobulin, lysozyme, etc.), lactoferrin and lipids (1\%), water, mucin, salt and proteins. $\operatorname{IgA}$, $\operatorname{IgE}$, and $\operatorname{IgG}$ are also present in mucous secretions. The $\mathrm{pH}$ of nasal secretions varies from 5 to 6.5 . The area of the olfactory zone is $10 \mathrm{~cm}^{2}$. Olfactory receptor neurons are bipolar neurons that carry information from the epithelium to the sense of smell. 


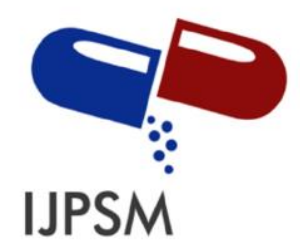

Nikam Supriya S. et al, International Journal of Pharmaceutical Sciences and Medicine (IJPSM), Vol.6 Issue. 7, July- 2021, pg. 61-80

ISSN: 2519-9889

Impact Factor: 3.426

\section{Transport of Active Moieties/ Nanocarrier from Nose to Brain ${ }^{[2]}$}

In 1909, scientist Paul Ehrlich led the era of targeted delivery when he invented a drug delivery mechanism that directly targets diseased cells. Since then, many transporters have been used to deliver drugs to target organs and tissues. Based on recent studies, the nasal brain pathway is a potential pathway that delivers therapeutic nanotransporters directly to the brain via a non-invasive pathway that bypasses the BBB. The pathways of the transport mechanism include three different pathways, including the sensory pathway, the trigeminal nerve pathway, and the systemic pathway, the outline of which is shown in Figure 2.

The nasal mechanism of the brain has been extensively studied to develop formulations that improve brain targeting.

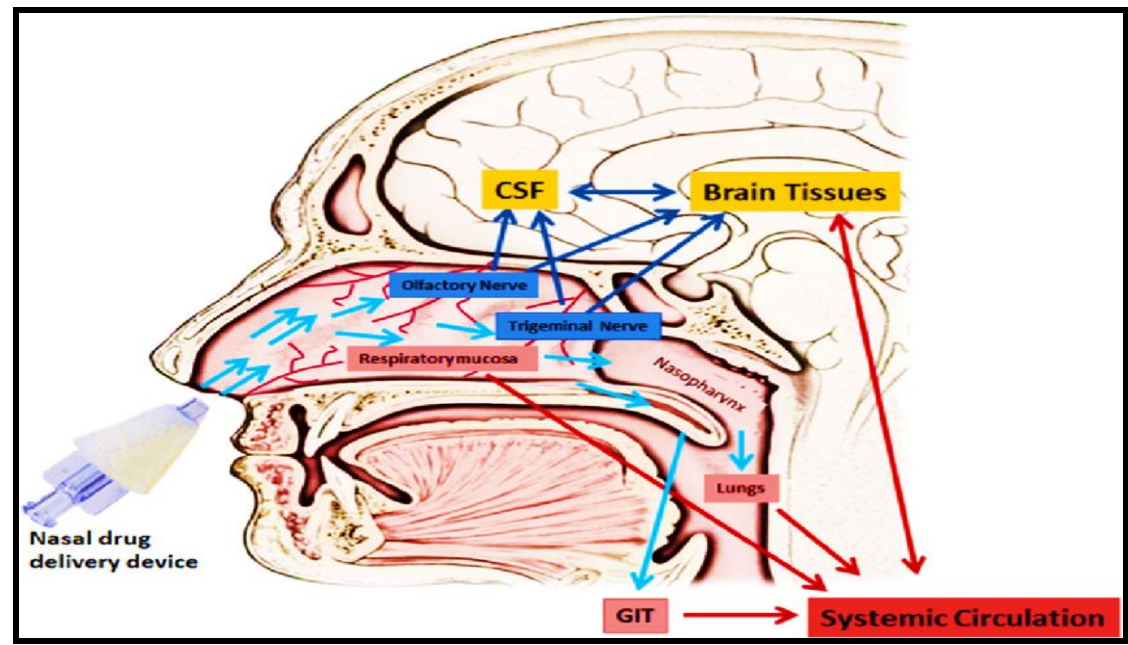

Figure 2. Overview of three different pathways after nasal administration.

\section{Mechanism of Pathways}

\section{Olfactory pathway ${ }^{[2]}$}

When delivered through the nose, the therapeutic segment reaches the olfactory mucosa / olfactory epithelium shown in Figure 3. The olfactory mucosa contains olfactory receptive neurons that help conduct and occur in the cilia, which are the ends of the olfactory receptive neurons. Molecules reach the olfactory receptor neurons via cellular / transcellular mechanisms. The integrity of the nasal epithelium, as well as tight junctions, desmoma, adhesive junctions, and intercellular spaces allow compounds to enter by intracellular transport. The neural pathway is thought to be the stage that defines the nasal glands. The fragments of the drug that travel along the axons, through the nerve 


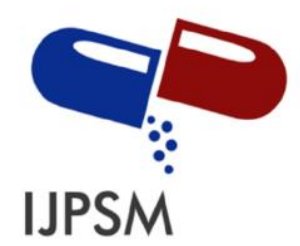

Nikam Supriya S. et al, International Journal of Pharmaceutical Sciences and Medicine (IJPSM), Vol.6 Issue. 7, July- 2021, pg. 61-80

ISSN: 2519-9889

Impact Factor: 3.426

bundles, through the lamina cribrosa and into the olfactory bulb appear to actually be on the surface of the brain. From the olfactory nerve, the treatment roots can reach the cerebrospinal fluid (CSF) and smell. The drug can be delivered from the cerebrospinal fluid to the brain by mixing with the interstitial fluid in the brain. After intranasal administration, it only takes a few minutes to reach the brain via sensory transport. Intraneuronal and extraneuronal pathways are two different transmission pathways from sensory neurons to the brain. The intraneuronal pathway is involved in axial transport and the active part takes hours or even days to reach different parts of the brain. By the extra-nervous ways, there is transport through the extra-nervous ducts; it only takes a few minutes for the active part to reach the brain directly and the sensory nerve pathways to reach deeper areas of the brain such as the cerebral cortex, cerebellum.

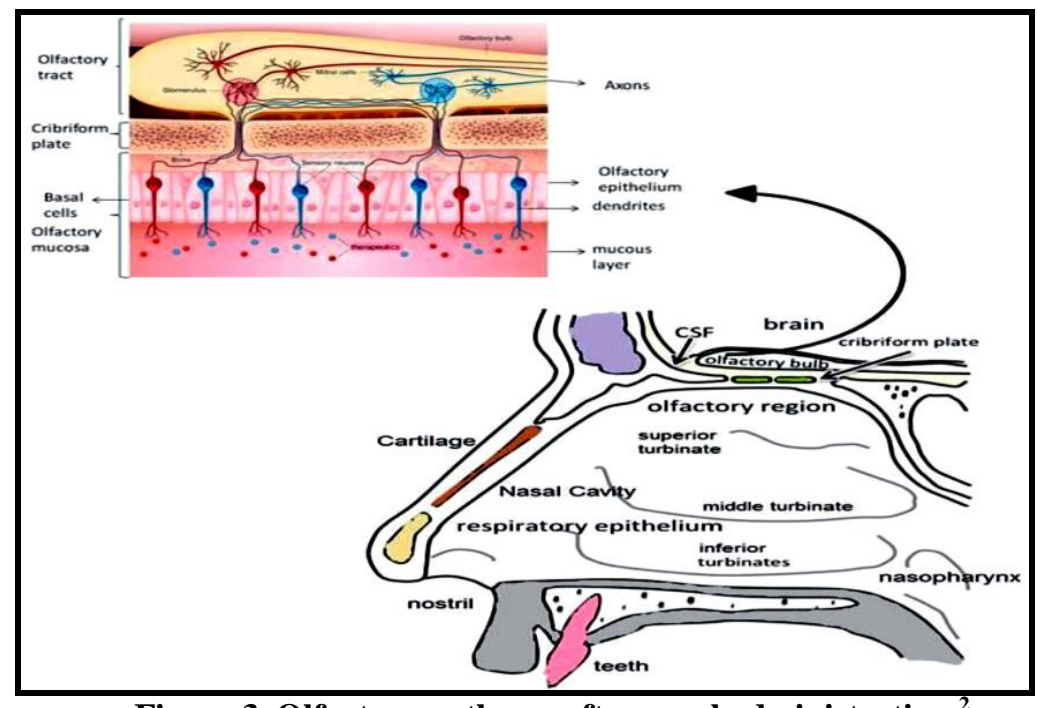

Figure 3. Olfactory pathway after nasal administration. ${ }^{2}$

\section{Trigeminal pathway ${ }^{[2]}$}

The trigeminal nerve connects to the tail of the brain, such as the spinal cord, long spinal cord, and joints. Drugs are transported from the nose through the trigeminal nerve by intracellular or axial transport or by intracellular processes. The trigeminal nerve is the fifth largest cranial nerve and is made up of three branches: the basal nerve, the maxillary nerve, and the mandibular nerve. The delivery of the drug by the three leading ophthalmologists and from the maxillary branch to the nasal brain is important, and the neurons of these branches pass directly through the nasal mucosa. Part of the trigeminal nerve ends at the level of the olfactory bulb. The dorsal part of the nasal mucosa and the trigeminal branch of the anterior nasal cavity resemble the upper branch of the turbine of the nasal mucosa. When the compounds pass through the mucous membranes of the nasal cavity, they reach the trigeminal nerve branches of the sensory and respiratory regions and pass through the brainstem for axial transport. Some of the trigeminal nerves pass 


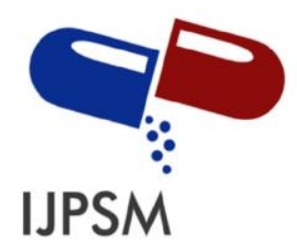

Nikam Supriya S. et al, International Journal of Pharmaceutical Sciences and Medicine (IJPSM), Vol.6 Issue. 7, July- 2021, pg. 61-80

ISSN: 2519-9889

Impact Factor: 3.426

through the stroma and may also be involved in healing the nasal passages to the forebrain. It has been reported that after intranasal injection of insulin-like growth factor I (IGFI), it rapidly reaches the brain via the trigeminal nerve. Nasal medications are absorbed through the nose through the mucus. This is the first step in the absorption process. After passing through mucus, several mechanisms are involved in mucosal transport, intracellular transport, transcellular transport, vector mediation, receptor mediation, and cell migration. The intracellular pathway is the transport of molecules between cells. The transcellular pathway refers to cellular drug delivery, which can occur via vector-mediated transport or intracellular processes. In the transcellular pathway, the adsorbed transcellular mechanism involves the transport of macromolecules. This is a process that involves the interaction of blood ligands with the cell surface. This type of interaction can result from an electrostatic interaction between a positively charged ligand, such as a protein or macromolecule, and a negatively charged membrane. Nanoparticles and some compounds undergo cell permeation. Transporter-mediated uptake has been suggested to be mediated by organic cation transporters, glycoproteins, amino acid transporters, and dopamine transporters that function as molecular transporters in the nasal mucosa.

\section{Systemic Pathway ${ }^{[2]}$}

The application of the drug from the nasal cavity to the brain is also done through the bloodstream. Due to the high vascular distribution of the respiratory epithelium, the olfactory mucosa of the drug is submerged in the systemic circulation. The airways are composed of a fusion of continuous endothelium and fused endothelium, and large and small molecules pass through the bloodstream and are transported to the CNS via the BBB. Lipophilic small molecules are easier to enter the bloodstream and pass through the BBB than high molecular weight, hydrophilic molecules. The active part is distributed throughout the circulatory system. It enters the blood vessels of the nose and is quickly transferred to the brain and spinal cord through the bloodstream of the carotid arteries. This is a process known as retrosexual exchange.

\section{Factors Affecting Nasal Drug Absorption:}

The physicochemical properties of the drug, the clearance of the nasal mucus and the activator of nasal absorption are the main factors affecting the absorption of the drug through the nasal mucosa. One of the biggest limitations of nasal drops is the inadequate absorption of the drug through the nasal passages. Some promising drug candidates are not available intranasally because researchers are trying to promote drug absorption through the nasal passages and they are not absorbed enough to produce a therapeutic effect, various factors are shown in the Fig. 3. 


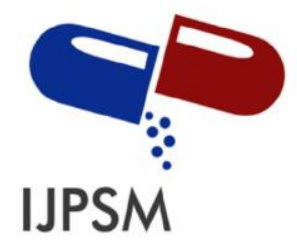

Nikam Supriya S. et al, International Journal of Pharmaceutical Sciences and Medicine (IJPSM),

Vol.6 Issue. 7, July- 2021, pg. 61-80

ISSN: 2519-9889

Impact Factor: 3.426
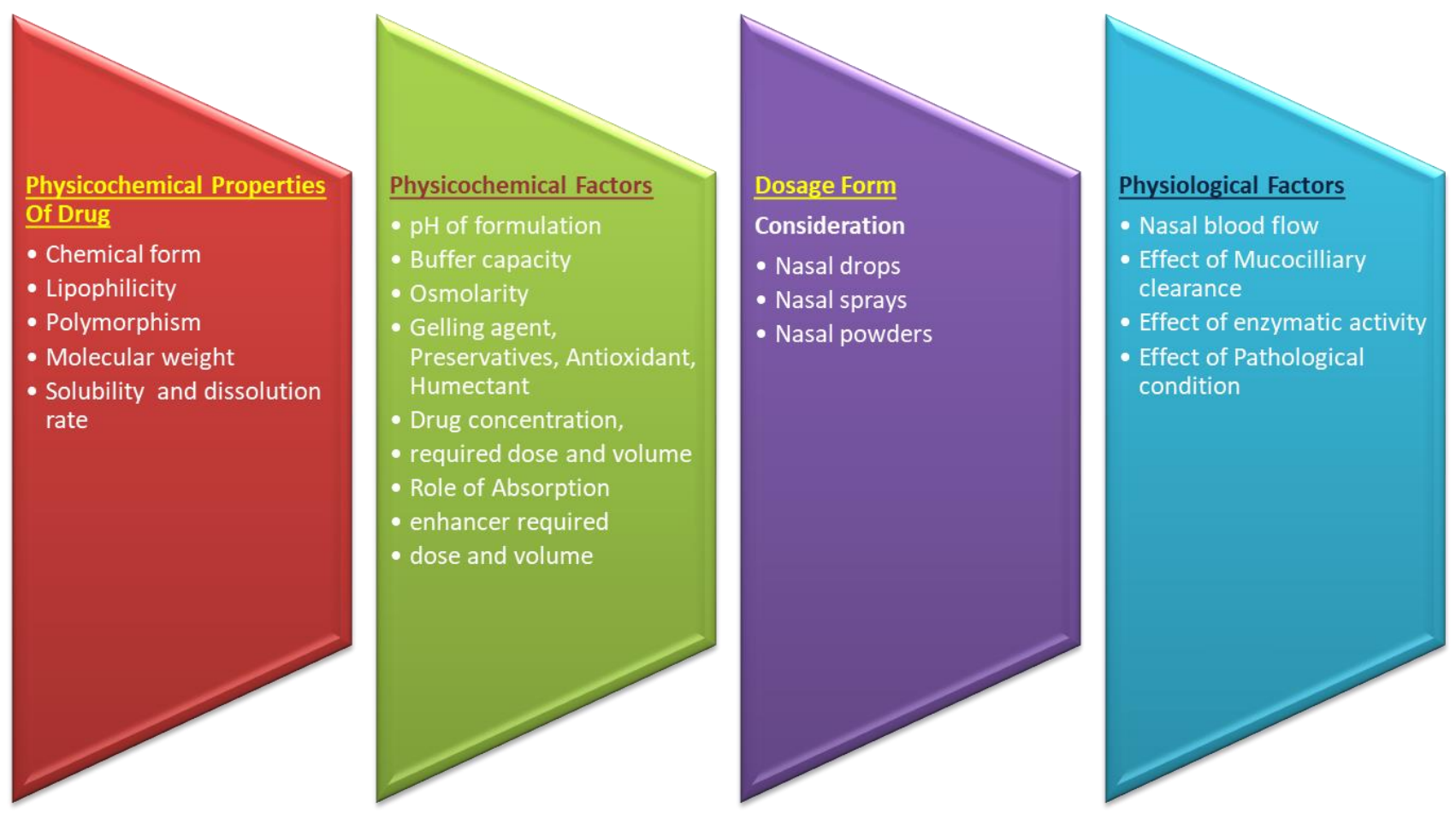

Figure.3 Factors affecting nasal drug absorption

Nano/ lipid based carriers (versatility of different carriers for drug delivery/ targeting) ${ }^{[17][18][19][20][21]}$

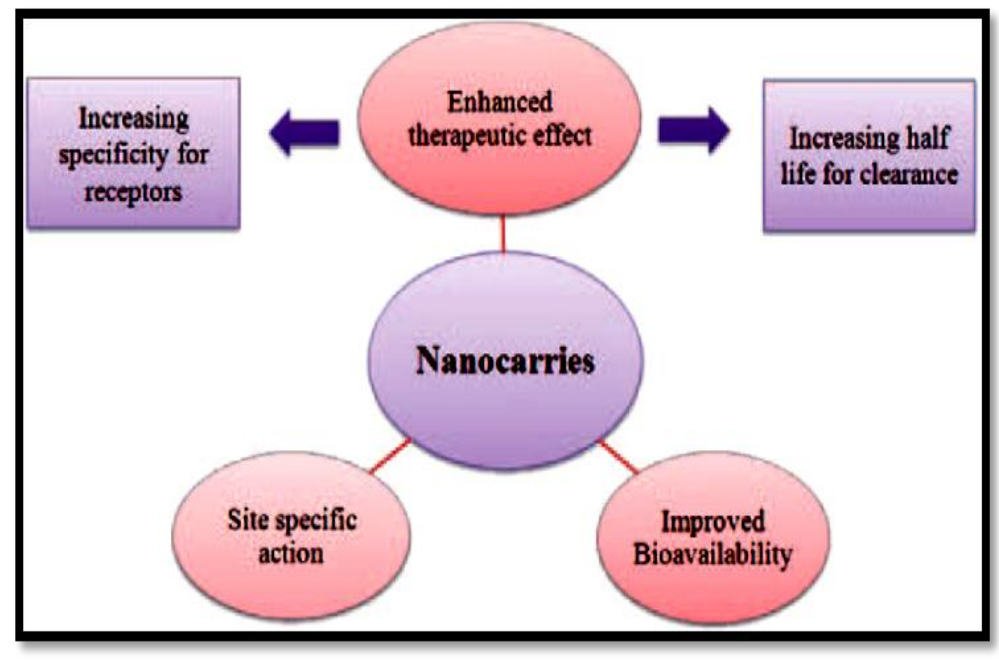

Figure 4. Important feature of nanocarrier. 


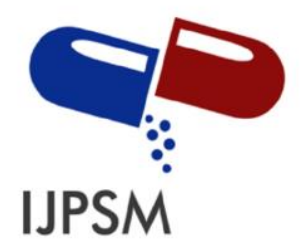

Nikam Supriya S. et al, International Journal of Pharmaceutical Sciences and Medicine (IJPSM), Vol.6 Issue. 7, July- 2021, pg. 61-80

ISSN: 2519-9889

Impact Factor: 3.426

Continuing improvement in the pharmacological and therapeutic properties of drugs is achieving the revolution in novel drug delivery systems. A wide spectrum of therapeutic nanocarriers has been widely investigated to address this developing need. This article will review recent developments in the use of nanoparticles as drug delivery systems to treat an extensive variety of diseases. Finally, we will introduce challenges and future nanotechnology strategies to overcome limitations in this field. Drug targeting can be defined as the ability to direct a therapeutic agent specifically to desired site of action with little or no interaction with non-target tissue. Paul Ehrlich, in 1909, initiated the era of development for targeted delivery when he envisaged a drug delivery mechanism that would target directly to diseased cell. Since then, numbers of carriers were utilized to carry drug at the target organ or tissue. The concept of targeted drug delivery is designed for attempting to focus on the drug in the tissues of interest while reducing the relative concentration of the medication in the remaining tissues. Currently, Nano medicine is dominated by drug delivery systems, accounting for more than $75 \%$ of total sale. Due to the advancements of nanotechnology, Nano medicine has grew well as nanostructures could serve as a delivery vehicle for encapsulating or attaching therapeutic moieties for targeted and controlled drug delivery of drugs, genes and growth factors. Such nanodrugs have higher oral bioavailability, prolonged stay in the bloodstream and fewer side effects. They achieve such effects by varying intrinsic properties of drug molecules such as solubility, drug release profiles, diffusivity, bioavailability and immunogenicity In the core nanotechnology, the term 'nano' is used for particles which are less than $100 \mathrm{~nm}$ whereas nanocarriers are termed as particles lying between few nanometers to $1000 \mathrm{~nm}$ and rather than its small dimensions which favour better penetrability and high surface area, properties and characteristics of such nanomaterial's are distinguishably different that of bulk materials. Lipid carriers can be characterised into various types depending on their method of preparation and physicochemical characteristics. They include liposomes, niosomes, solid lipid nanoparticles (SLN) and nanostructured lipid carriers (NLC). Nanocarriers having different aspects as shown in above figure 4.

\section{Structures of NLCs ${ }^{[22]}$}

When SLNs are prepared from solid lipids, the matrix tends to form a relatively perfect crystal lattice, leaving limited space to accommodate the active ingredients Figure 5. shows the proposed structure of the inner cores of SLNs. In contrast, the employment of a lipid blend including solid and liquid forms can distort the production of a perfect crystal. The particle matrix contains imperfections, providing space to accommodate the drug molecules in amorphous clusters Figure 5. It has also been proposed that NLCs are composed of oily droplets embedded in a solid lipid matrix. The morphology of particles of NLCs is not essential spherical. 


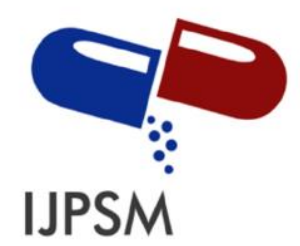

Nikam Supriya S. et al, International Journal of Pharmaceutical Sciences and Medicine (IJPSM), Vol.6 Issue. 7, July- 2021, pg. 61-80

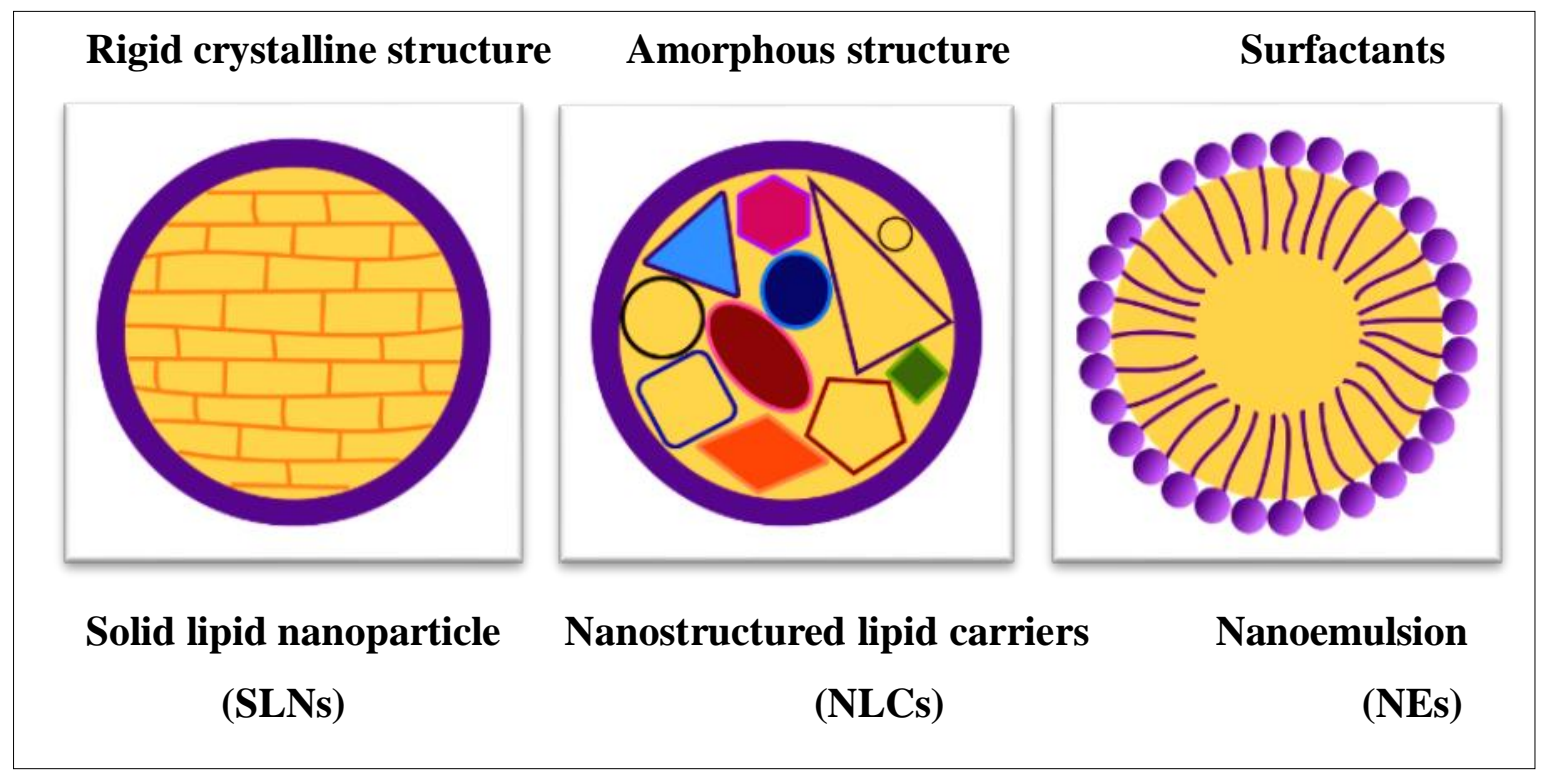

Figure 5. Different types of Nanoparticles.

\section{Types of $N L C^{[23]}$}

Different types of NLCs can be obtained on the basis of way of production and the composition of the lipid blend. The basic idea is that by giving the lipid matrix a nanostructure, the pay-load for active compounds is increased and expulsion of the compound during storage is avoided. The three types can be summarized as follows:

$>$ The imperfect type

$>$ The amorphous type

$>$ The multiple type

\section{The imperfect type:}

It can also be stated as imperfect type. Replacement of a fraction of solid lipid by liquid lipid/oil causes formation of imperfect crystal lattice/matrix. This phenomenon shows availability of more space for accommodation of drug and allows higher drug loading. Formation of imperfect crystal core gives more space for drug incorporation, avoiding formation of highly structured or ordered matrix which would have expelled drug out of the core. 


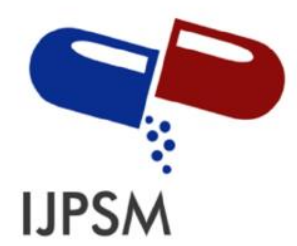

Nikam Supriya S. et al, International Journal of Pharmaceutical Sciences and Medicine (IJPSM), Vol.6 Issue. 7, July- 2021, pg. 61-80

ISSN: 2519-9889

Impact Factor: 3.426

\section{The amorphous type:}

This is also known as amorphous/ structureless type. Use of solid lipids which remain in $\alpha$ polymorph after solidification and storage along with liquid lipids tend to form amorphous core. This is advantageous over type I NLCs as no crystallization occurs and drug remains embedded in amorphous matrix. The $\beta$ polymorph of solid lipids develop crystalline structured matrix.

\section{The multiple type:}

This is multiple type and developed from the concept of w/o/w emulsion. It is basically oil-in-solid or fat-in-water type NLC, which can be developed only by phase separation technique. When drug shows higher solubility in oil, this approach can be utilized in formulation of NLCs in order to improve drug loading capacity and stability. Tiny droplets of oil are dispersed uniformly in solid lipid matrix and this system is dispersed in the aqueous medium.

\section{Advantages of NLC's:}

$>$ Better physical stability

$>$ Ease of preparation and scale-up

$>$ Increased dispersability in an aqueous medium

$>$ High entrapment of lipophilic drugs and hydrophilic drugs

$>$ Controlled particle size

$>$ An advanced and efficient carrier system in particular for substances

$>$ Increase of skin obstruction

$>$ Extended release of the drug

$>$ Improve benefit or risk ratio

$>$ Increase of skin hydration as well as elasticity

$>$ Lipid components have an approved status or are excipients used in commercially available topical cosmetic or pharmaceutical preparations

$>$ Small size of the lipid particles confirms close contact to the stratum corneum thus enhancing drug penetration into the mucosa or skin

$>$ These carriers are highly efficient systems due to their solid lipid matrices, which are also generally recognized as safe or have a regulatory accepted status 


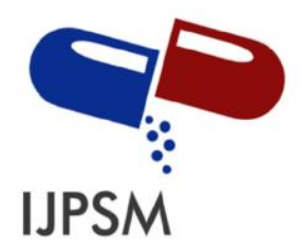

Nikam Supriya S. et al, International Journal of Pharmaceutical Sciences and Medicine (IJPSM), Vol.6 Issue. 7, July- 2021, pg. 61-80

ISSN: 2519-9889

Impact Factor: 3.426

\section{Limitations of Lipid Nanoparticles:}

Despite the great potential of NLCs in targeted delivery, they face certain limitations like: Cytotoxic effects related to the nature of matrix and concentration, irritation and sensitizing action of some surfactants and lack of sufficient preclinical and clinical studies with these nanoparticles in case of bone repair.

\section{Composition of NLC ${ }^{[25]}$}

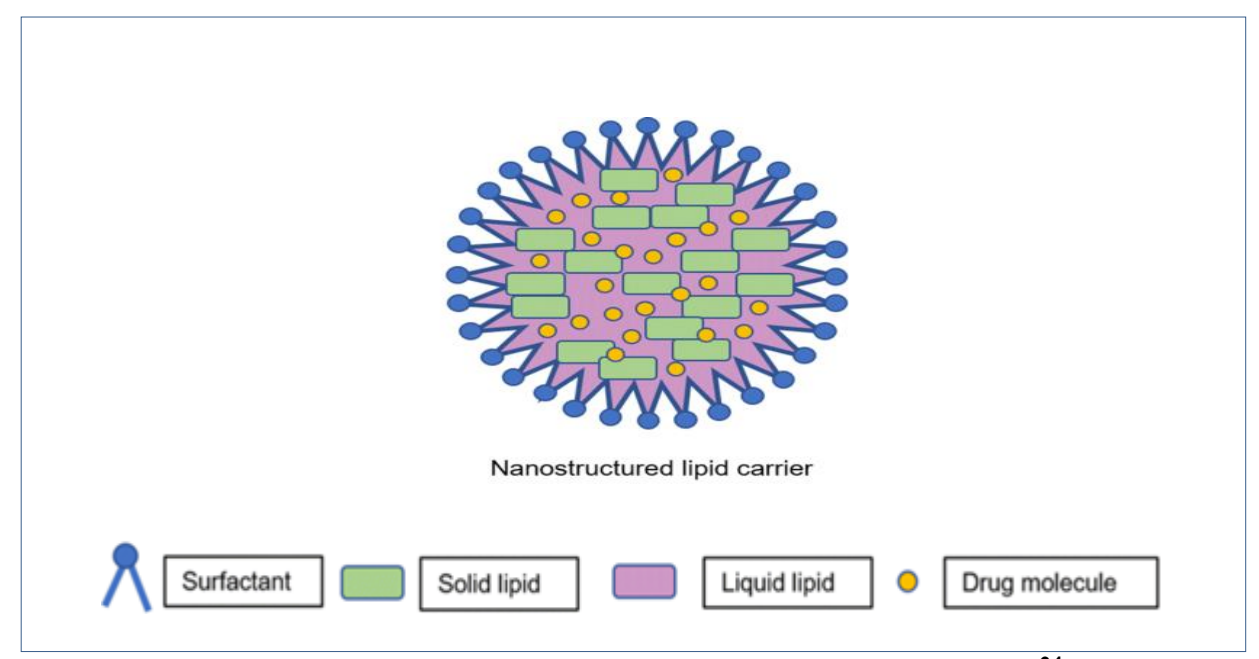

Figure 6. Composition of nanostructured lipid carriers. ${ }^{24}$

\section{Solid lipids:}

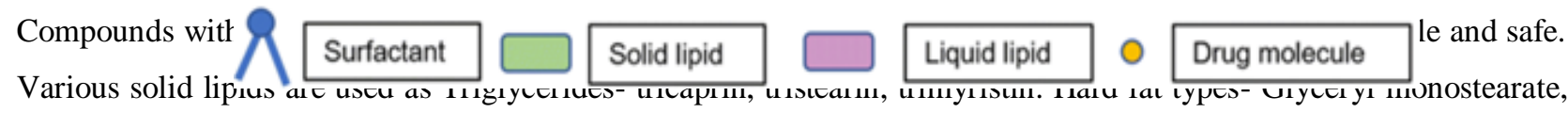
stearic acid, cetyl alcohol.

\section{Liquid lipids:}

The liquid lipids used are digestable oils from natural sources. It must have well tolerated GRAS status and also accepted for human use. Natural and synthetic oils are used such as castor oil, mustard oil, codliver oil, medium chain triglycerides and oleic acid. 


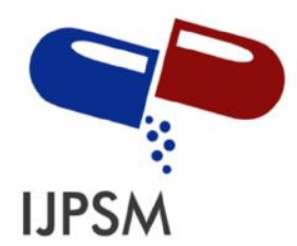

Nikam Supriya S. et al, International Journal of Pharmaceutical Sciences and Medicine (IJPSM), Vol.6 Issue. 7, July- 2021, pg. 61-80

ISSN: 2519-9889

Impact Factor: 3.426

\section{Aqueous surfactant:}

Surfactants are also called emulsifying agents which lower the interfacial tension between two immiscible liquids or components. Hydrophilic surfactants are mostly used. Lipophilic or ampiphillic emulsifiers are used for the fabrication of NLCs. Eg. Tween-80 and Pluronic F-68. Co-emulsifier: Soya lecithin, phosphatidylcholine, egg lecithin.

\section{Methods of Lipid Nanoparticles Preparation [17][21][27][28]}

Lipid nanoparticles could be prepared by various methods like hot and cold high pressure homogenization, solvent evaporation, melt emulsification, microemulsion formation technique, and ultrasonic solvent emulsification etc. for large-scale productions of lipid nanoparticles are mainly obtained by high pressure homogenization technique.

\section{Hot homogenization techniques:}

The hot homogenization method consists of evenly dispersing the molten mixture of lipid and drug under vigorous stirring in an aqueous solution containing surfactant that is previously heated to the same temperature. After this process, the pre-emulsion so formed is subjected to homogenization process which results in the production of hot oilin-water nanoemulsion. This Nano emulsion when subjected to cooling results in lipid recrystallization and production of lipid NPs (NLCs or SLNs).

\section{Cold high pressure homogenization:}

This method is suitable for heat-labile drugs or hydrophilic drugs. The lipid and drug are melted together and rapidly cooled under liquid nitrogen forming solid lipid micro particles, a presuspension is formed by homogenization of the particles in a cold surfactant solution. The pre-suspension is then further homogenized in a high pressure homogenization at or below room temperature at predetermined homogenization condition to produce NLC. In this both high pressure homogenization techniques are suitable for processing lipid concentrations of upto $40 \%$ and generally they yield very narrow particle size distributions. Cold homogenisation minimises the thermal exposure of the sample.

\section{Melt emulsification technique:}

The lipid mix, surfactant and drug were melted and dispersed in hot aqueous surfactant solution at same temperature using mild stirring to form primary emulsion. The warm primary emulsion was subjected to lab ultrasonic cell pulverizer to obtain nano-emulsion whish was rapidly cooled by immersing beaker in ice-cold water and stirred to obtain uniform NLC dispersion. 


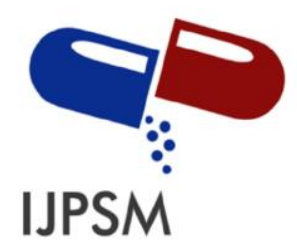

Nikam Supriya S. et al, International Journal of Pharmaceutical Sciences and Medicine (IJPSM), Vol.6 Issue. 7, July- 2021, pg. 61-80

ISSN: 2519-9889

Impact Factor: 3.426

\section{Solvent evaporation technique:}

This technique involves the addition of LM and drug in organic solvent which is then emulsified in an aqueous phase. After evaporation of the solvent the lipid precipitates forming NLC.

\section{Microemulsion technique:}

In this technique, the lipids are melted and drug is incorporated in molten lipid. A mixture of water, co-surfactant(s) and the surfactant is heated to the same temperature as the lipids and added under mild stirring to the lipid melt. A transparent, thermodynamically stable system is formed when the compounds are mixed in the correct ratios for microemulsion formation. Thus the microemulsion is the basis for the formation of nanoparticles of a requisite size. This microemulsion is then dispersed in a cold aqueous medium under mild mechanical mixing of hot microemulsion with water in a ratio in the range 1:25-1:50. This dispersion in cold aqueous medium leads to rapid recrystallization of the oil droplets. Surfactants and co-surfactants include lecithin; biliar salts alongwith alcohols such as butanol. Excipients such as butanol are less commonly used due to their regulatory aspects. The microemulsion is prepared in a large, temperature- controlled tank and then pumped from this tank into a cold water tank for the precipitation step.

\section{Application Nanostructured Lipid Carriers for Nose to Brain Targeting [21][27][29]}

A new generation of nanostructured lipid carriers (NLCs) containing of a lipid matrix with a special nanostructure has been developed. This nanostructure improves drug loading and definitely incorporates the drug during storage also These NLCs can be made by high-pressure homogenization and the process can be altered to yield lipid particle dispersions with solid contents from $30-80 \%$.

As the increasing incidences of central nervous system diseases such as Alzheimer's disease, Parkinson's disease, epilepsy, brain infections, stroke, brain tumor, epilepsy, drug delivery to brain possess a great challenge as almost $98 \%$ of the newly exposed therapeutics are unable to cross BBB

Nanotechnology has broadened the medical tools and treatments available to clinicians, which is an application of the field of nanotechnology in medicine, has provided precise solutions for disease diagnosis, prevention while conventional dosage forms used for treating the CNS disorders lack specificity and target-ability which culminates in lower therapeutic concentrations at the site of action and undesirable interactions with adjacent tissues (toxicity), influencing therapeutic. Efflux transporters present in the brain further limit the brain bioavailable fraction of loaded CNS active drugs by returning them into systemic circulation. Nanotechnology principles such as, SLN and NLC followed by their chemical or physical modifications, provide some unique dynamic systems that appear to be very beneficial to improve localized delivery of drug into brain by crossing BBB thereby mitigate neurological disorders. Brain targeting increases the cerebrospinal fluid concentration of the drug as well as reduces the frequency of dosing and side effects. The main advantage of this administration route is avoidance of first pass metabolism and rapid onset 


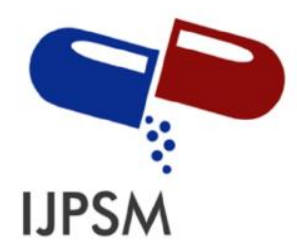

Nikam Supriya S. et al, International Journal of Pharmaceutical Sciences and Medicine (IJPSM), Vol.6 Issue. 7, July- 2021, pg. 61-80

ISSN: 2519-9889

Impact Factor: 3.426

of action as compared to oral administration. NLC of this generation are considered to be one of the major strategies for drug delivery without any modification to the drug molecule because of their rapid uptake by the brain, bioacceptability and biodegradability. Further, the possibility in scale-up and absence of burst effect make them more promising carriers for drug delivery. NLC further improved the intranasal drug delivery of duloxetine in the brain for the treatment of major depressive disorder. Asenapine-NLC maleate to improve the bioavailability and enhance Asenapine in brain. In Bromocriptin loaded NLCs, the In- vivo results displayed bromocriptine NLCs have rapid onset of action and longer duration and higher brain levels as compared to that of solution, entrapment efficiency was also improved. The results of the study also suggest that CRM-NLC is a promising drug delivery system for brain cancer therapy.

\section{Current development of NLCs in brain targeting through nasal route :}

NLCs can generally be applied where solid nanoparticles possess advantages for the delivery of drugs. Major application areas in pharmaceutics are topical drug delivery, oral and parenteral administration. They also have applications in cosmetics, food and agricultural products.

To form resveratrol nanostructured in situ gel for the treatment of Alzheimer's disease. Resveratrol loaded lipid carrier was prepared by melt emulsification-probe sonication method, and the final product was evaluated for particle size $(132 \pm 11.90 \mathrm{~nm})$, polydispersity index $(0.209 \pm 0.005)$, zeta potential $(-23 \pm 3.79 \mathrm{mV})$, drug loading $(9.26 \pm 3.79 \%)$, and entrapment efficiency $(74 \pm 11.40 \%)$ as studied in Rajput A, et al., 2018. Incorporation of the resveratrol nanostructured lipid carrier in gellan gum and xanthan gum, in situ gel was formulated and characterized. The optimized in situ gel shows fivefold higher permeation across the nasal mucosa as compared to resveratrol suspensionbased in situ gel. Optimized in situ gel was estimated using in vivo pharmacodynamic study by the scopolamineinduced amnesia model in rats using Morris Water Maze test. It showed significant development in memory function in rats treated with optimized in situ gel as compared to orally administered resveratrol suspension. The improved permeation across nasal mucosa and enhanced memory function suggest that the resveratrol nanostructured lipid carrier-based in situ gel could be an effective and promising approach for the treatment of Alzheimer's disease.

PIO treatment was found to recover AD significantly in the preclinical models, the poor BBB permeability and serious peripheral side effects limited its success in the clinical trials. This study is given in Jojo M, et al., 2019. The aim of the present study was to formulate and optimize intranasal (IN) nano lipid carriers (NLC) of PIO for its targeted delivery to the brain, Box-Behnken design was employed to optimize the effect of three independent variables on two dependent variables. The optimized formulation had a particle size (PS) of $211.4 \pm 3.54 \mathrm{~nm}$ and zeta potential of (ZP) of $14.9 \pm 1.09 \mathrm{mv}$. The polydispersibility index (PDI) and entrapment efficiency (EE) was found to be $0.257 \pm 0.108$ and $70.18 \pm 4.5 \%$ respectively. Storage stability studies performed has confirmed the stability of NLCs at $4 \mathrm{C}$ and 25 C. The in-vitro drug release study has showed a sustained release of drug from the NLC. The formulation was 


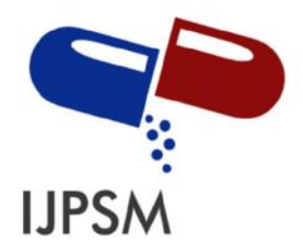

Nikam Supriya S. et al, International Journal of Pharmaceutical Sciences and Medicine (IJPSM), Vol.6 Issue. 7, July- 2021, pg. 61-80

ISSN: 2519-9889

Impact Factor: 3.426

observed to improve the nasal permeability of PIO ex-vivo significantly. Toxicity studies were performed to confirm the safety of formulation for the in-vivo administration. In-vivo biodistribution study in rats has shown a direct transport of drug from the nose to brain from the IN-NLC.

Explored brain targeting efficiency of estradiol (E(2))-loaded chitosan nanoparticles. Nano formulation was organized by ionic gelation of chitosan with tripolyphosphate anions. As per study given in Wang X, et al., 2008 the CS-NPs had a mean size of $269.3+/-31.6 \mathrm{~nm}, \mathrm{ZP}$ of $+25.4 \mathrm{mV}$, and of $64.7 \%$. CS-NPs were administered to male Wister rats either intranasally or intravenously at the dose of $0.48 \mathrm{mg} / \mathrm{kg}$. The plasma levels achieved following intranasal administration $(32.7 \pm 10.1 \mathrm{ng} / \mathrm{ml})$ and tmax $(28 \pm 4.5 \mathrm{~min})$ were significantly lower than those after intravenous administration $(151.4 \pm 28.2 \mathrm{ng} / \mathrm{ml})$, while CSF concentrations achieved after intranasal administration $(76.4 \pm 14.0$ $\mathrm{ng} / \mathrm{ml}$; tmax $28 \pm 17.9 \mathrm{~min})$ were significantly higher than those after intravenous administration $(29.5 \pm 7.4 \mathrm{ng} / \mathrm{ml}$; tmax $60 \mathrm{~min}$ ). The drug targeting index of nasal route was $3.2 \%$ of drug targeting was $68.4 \%$; indicate a direct transport of formulation from nose to brain.

Investigate the influence of the nanocarrier surface charge on brain delivery of a model hydrophilic drug via the nasal route. Anionic and cationic NLC were prepared and optimized for their particle size and zeta potential. In the investigation study of Gabal YM et al., 2014 the optimum particles were incorporated in poloxamer in situ gels and their in vivo behaviour was studied in the plasma and brain after administration to rats. Optimum anionic and cationic NLCs of size $<200 \mathrm{~nm}$ and absolute zeta potential value of $-34 \mathrm{mV}$ were obtained. Toxicity study revealed mild to moderate reversible inflammation of the nasal epithelium in rats treated with the anionic NLCs, and destruction of the lining mucosal nasal epithelium in rats treated with the cationic NLCs. The absolute bioavailability of both drug loaded anionic and cationic NLCs in situ gels was enhanced compared to that of the intranasal solution (IN) of the drug with values of $44 \%$ and $77.3 \%$, respectively. Cationic NLCs in situ gel showed a non-significant greater Cmax in the brain compared to the anionic NLCs in situ gel, Anionic NLCs in situ gel gave highest drug targeting efficiency in the brain (DTE\%) with a value of 158.5 which is nearly 1.2 times that of the cationic NLCs in situ gel.

\section{Conclusion:}

Alzheimer disease is the most common cause of dementia, still now there is no any treatment on Alzheimer disease. On early stage of Alzheimer there is not any diagnosis of the disease so it is necessary to diagnose in early stages. Now a day's nanotechnology achieves more success for treatment of disease due to its versatile properties. In this article the use of nanotechnology carrier that is nanostructured lipid carriers for Alzheimer disease. In which the route chosen is nasal route because of it more absorption capacity through nasal mucosa is high and it helps to transfer the drug moiety from nose to brain directly from the pathway mechanisms. So this approach is best for the nose to brain 


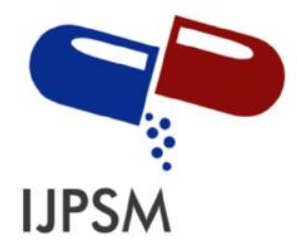

Nikam Supriya S. et al, International Journal of Pharmaceutical Sciences and Medicine (IJPSM), Vol.6 Issue. 7, July- 2021, pg. 61-80

ISSN: 2519-9889

Impact Factor: 3.426

targeting directly through olfactory and trigeminal pathway. The different approaches of nanocarrier, types, different preparation methods, advantages and disadvantages as well. Different scientists showed the growth of nanotechnology with different drugs and disease.

\section{References}

[1]. Charlton ST, Whetstone J, Fayinka ST, Read KD, Illum L, Davis SS. Evaluation of direct transport pathways of glycine receptor antagonists and an angiotensin antagonist from the nasal cavity to the central nervous system in the rat model. Pharmaceutical research. 2008 Jul;25(7):1531-43.

[2]. Selvaraj K, Gowthamarajan K, Karri VV. Nose to brain transport pathways an overview: Potential of nanostructured lipid carriers in nose to brain targeting. Artificial cells, nanomedicine, and biotechnology. 2018 Nov 17;46(8):2088-95.

[3]. Lawther BK, Kumar S, Krovvidi H. Blood-brain barrier. Continuing Education in Anaesthesia, Critical Care \& Pain. 2011 Aug 1;11(4):128-32.

[4]. Illum L. Is nose- to- brain transport of drugs in man a reality?. Journal of Pharmacy and Pharmacology. 2004 Jan;56(1):3-17.

[5]. Illum L. Nasal drug delivery_-possibilities, problems and solutions. Journal of controlled release. 2003 Feb 21;87(1-3):187-98.

[6]. Hussein NR, Omer HK, Elhissi AM, Ahmed W. Advances in nasal drug delivery systems. InAdvances in Medical and Surgical Engineering 2020 Jan 1 (pp. 279-311). Academic Press.

[7]. Mathison S, Nagilla R, Kompella UB. Nasal route for direct delivery of solutes to the central nervous system: fact or fiction?. Journal of drug targeting. 1998 Jan 1;5(6):415-41.

[8]. Tajes M, Ramos-Fernández E, Weng-Jiang X, Bosch-Morato M, Guivernau B, Eraso-Pichot A, Salvador B, Fernandez-Busquets X, Roquer J, Munoz FJ. The blood-brain barrier: structure, function and therapeutic approaches to cross it. Molecular membrane biology. 2014 Aug 1;31(5):152-67.

[9]. Agrawal M, Saraf S, Saraf S, Antimisiaris SG, Chougule MB, Shoyele SA, Alexander A. Nose-to-brain drug delivery: An update on clinical challenges and progress towards approval of anti-Alzheimer drugs. Journal of controlled release. 2018 Jul 10;281:139-77.

[10]. Kinney JW, Bemiller SM, Murtishaw AS, Leisgang AM, Salazar AM, Lamb BT. Inflammation as a central mechanism in Alzheimer's disease. Alzheimer's \& Dementia: Translational Research \& Clinical Interventions. 2018 Jan 1;4:575-90.

[11].Parihar MS, Hemnani T. Alzheimer's disease pathogenesis and therapeutic interventions. Journal of clinical neuroscience. 2004 Jun 1;11(5):456-67.

[12].Rajput A, Bariya A, Allam A, Othman S, Butani SB. In situ nanostructured hydrogel of resveratrol for brain targeting: in vitro-in vivo characterization. Drug delivery and translational research. 2018 Oct;8(5):1460-70.

[13]. Kumar A, Singh A. A review on Alzheimer's disease pathophysiology and its management: an update. Pharmacological reports. 2015 Apr 1;67(2):195-203.

[14].Savale S, Mahajan H. Nose to brain: A versatile mode of drug delivery system. Asian J. Biomater. Res. 2017;3:16-38

[15]. Costa C, Moreira JN, Amaral MH, Lobo JS, Silva AC. Nose-to-brain delivery of lipid-based nanosystems for epileptic seizures and anxiety crisis. Journal of Controlled Release. 2019 Feb 10;295:187-200.

[16].Patel Z, Patel B, Patel S, Pardeshi C. Nose to brain targeted drug delivery bypassing the blood-brain barrier: an overview. Drug Invention Today. 2012 Dec 1;4(12).

[17].Jaiswal P, Gidwani B, Vyas A. Nanostructured lipid carriers and their current application in targeted drug delivery. Artificial cells, nanomedicine, and biotechnology. 2016 Jan 2;44(1):27-40.

[18].AK M, Dubal AP, Mane PD, Deshmukh HA. RECENT TRENDS IN NIOSOMES AS NANOCARRIERS.

[19]. Bansal BK, Shakya V, Rewar S. A Novel Trend in Drug Delivery System. Asian Journal of Pharmaceutical Research and Development. 2014 Mar 1:85-90. 


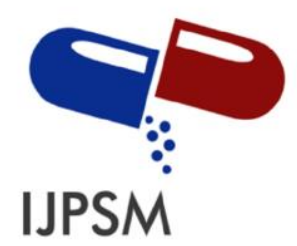

Nikam Supriya S. et al, International Journal of Pharmaceutical Sciences and Medicine (IJPSM), Vol.6 Issue. 7, July- 2021, pg. 61-80

ISSN: 2519-9889

Impact Factor: 3.426

[20].Bamrungsap S, Zhao Z, Chen T, Wang L, Li C, Fu T, Tan W. Nanotechnology in therapeutics: a focus on nanoparticles as a drug delivery system. Nanomedicine. 2012 Aug;7(8):1253-71.

[21].Pottoo FH, Sharma S, Javed MN, Barkat MA, Harshita, Alam MS, Naim MJ, Alam O, Ansari MA, Barreto GE, Ashraf GM. Lipid-based nanoformulations in the treatment of neurological disorders. Drug metabolism reviews. 2020 Jan 2;52(1):185-204.

[22].Fang CL, A Al-Suwayeh S, Fang JY. Nanostructured lipid carriers (NLCs) for drug delivery and targeting. Recent patents on nanotechnology. 2013 Jan 1;7(1):41-55.

[23].Salvi VR, Pawar P. Nanostructured lipid carriers (NLC) system: A novel drug targeting carrier. Journal of Drug Delivery Science and Technology. 2019 Jun 1;51:255-67.

[24].Subramaniam B, Siddik ZH, Nagoor NH. Optimization of nanostructured lipid carriers: Understanding the types, designs, and parameters in the process of formulations. Journal of Nanoparticle Research. 2020 Jun;22:1-29.

[25].Lokeshwar MR, Shah NK, Agarwal BR, Manglani MV, Sachdeva A, editors. Textbook of pediatric hematology and hemato-oncology. Jaypee Brothers Medical Publishers (P) Limited; 2016.

[26].Nayak AK, Alkahtani S, Hasnain MS. Biomedical Nanocomposites. Biomedical Composites: Perspectives and Applications. 2021:35.

[27].Karunakar G, Patel NP, Kamal SS. Nano structured lipid carrier based drug delivery system. J Chem Pharm Res. 2016;8(2):627-43.

[28].Natarajan J, Karri VV, Anindita D. Nanostructured lipid carrier (NLC): A promising drug delivery system. Global Journal of Nanomedicine. 2017;1(5):001-6.

[29].Radtke M, Müller RH. Nanostructured lipid drug carriers. New Drugs. 2001;2:48-52. 